\section{Boletín}

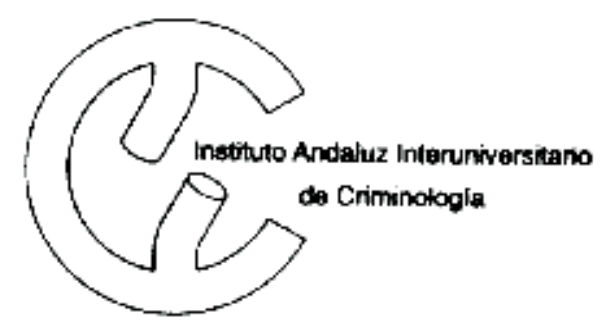

Criminológico

Directora: Elisa García España

Coordinadora: Susana García Ruiz

Publicado por la Sección de Málaga del IAIC

Edificio Institutos de Investigación, Universidad

de Málaga. Campus de Teatinos, 29071 MALAGA

Tel:(95) 2132325 - Fax: (95) 2132242

Depósitolegal: MA 857/1996 ISSN:1137-2427

www.uma.es/estudios/propias/criminologia

Recientemente el Consejo de universidades ha remitido al Ministerio de Educación una propuesta de Título oficial de Criminología, estructurado como una licenciatura de segundo ciclo. Se culminan así los accidentados esfuerzos del conjunto de Institutos de Criminología y Cursos de especialización de las diferentes universidades que, en colaboración con la Federación de asociaciones de criminólogos, hicieron una propuesta de directrices la cual, aunque con reformas de alguna importancia, ha sido finalmente asumida por el Consejo de universidades. De esta forma se va a dar carácter oficial a unos estudios con una notable tradición y consolidación en nuestro país, pero que nunca habían gozado de reconocimiento oficial.

Su existencia desde hace años como enseñanzas propias de las respectivas universidades, sin embargo, permite disponer de datos abundantes sobre las necesidades sociales a cubrir por tales estudios, sectores profesionales en ellos interesados y perfil de la demanda. El Instituto andaluz interuniversitario de Criminología viene impartiendo estas enseñanzas desde hace doce años, de manera homogénea en cinco universidades, y constituye el Instituto con mayor volumen de alumnado, profesorado y actividades en toda la nación. Consciente de ello, la sección de Málaga del Instituto ha creido conveniente poner a disposición del público una serie de datos significativos sobre la actual realidad de los estudios de Criminología en Andalucía, con la esperanza de que puedan ser útilies a la hora de estructurar e implantar estos estudios en el Estado. Con ello, por lo demás, los responsables de este Boletín continúan una práctica que ya tuvo un precedente en el Boletín número 30.

\title{
Instituto de Criminología de Málaga: 10 años de Docencia
}

\section{Introducción}

El Instituto

andaluz interuniversitario de Criminología fue creado por Decreto de la Junta de Andalucía 182/1990, de 5 de junio, al amparo de las posibilidades ofrecidas por el artículo 10 de la Ley de Reforma Universitaria. Estamos ante el primer Instituto con el carácter de «interuniversitario» aprobado en Andalucía, lo que significa que existe un único Instituto de Criminología para toda la Comunidad Autónoma con cinco secciones integradas en las Universidades de Cádiz, Córdoba, Granada, Málaga y Sevilla.

La sección de Málaga, desde su creación, viene desarrollando por un lado actividades de investigación, subvencionadas fundamentalmente por los programas gubernamentales europeos, nacionales o autonómicos, así como por otras entidades u organis- mos, y, por otro, de asesoramiento a las distintas instituciones que así lo soliciten o suscri-

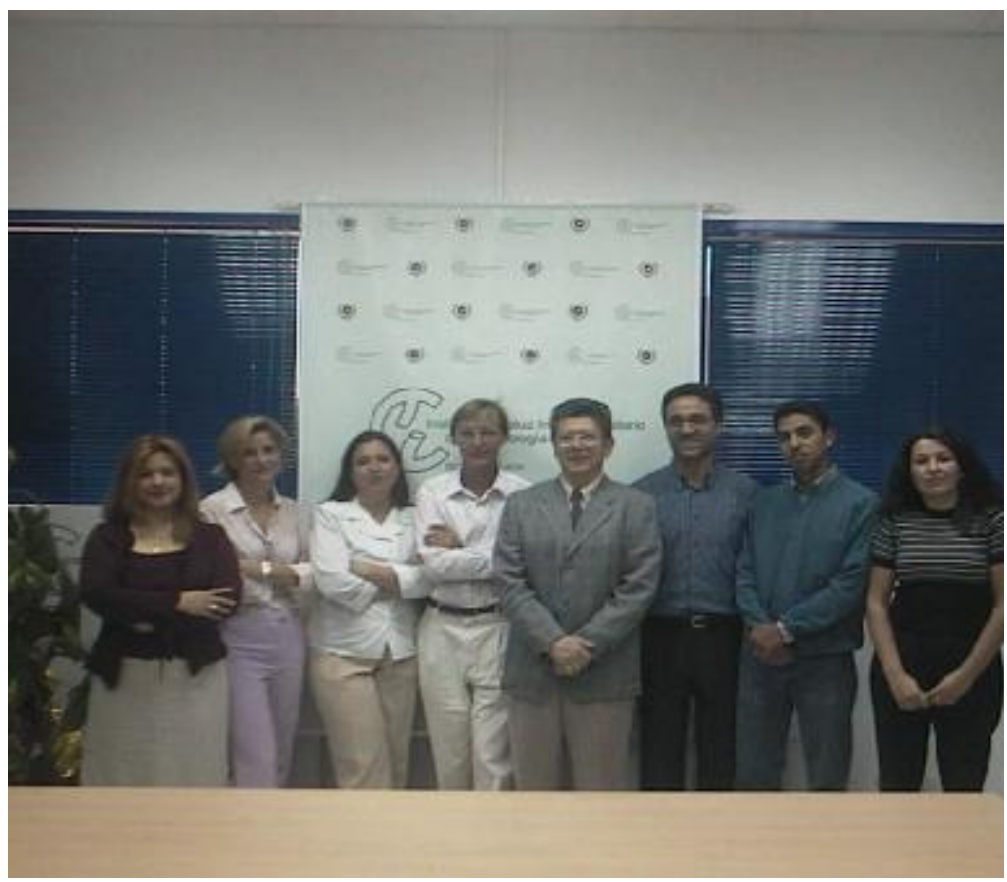

Autor:

Angel Serrano García

\section{Boletín Criminológico


Figura.1: «Evolución de los nuevos ingrsos en el I.A.I.C.»

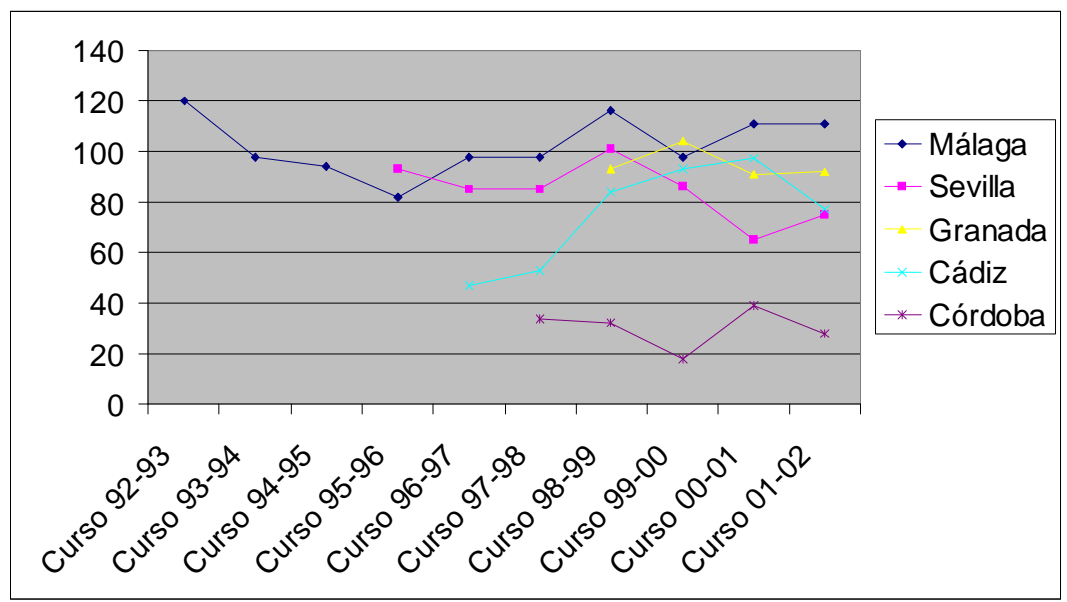

ban el correspondiente convenio de colaboración.

Fue en el curso 1992/1993 cuando la sección de Málaga comenzó la docencia en Criminología. Desde entonces se viene ofertando el Título de Experto Universitario en Criminología, cuyo plan de estudios se desarrolla durante 3 cursos académicos (780 horas) y va dirigido a universitarios que han superado el primer ciclo de estudios en cualquier carrera universitaria, así como a determinados profesionales especialmente dedicados a la prevención y lucha contra la delincuencia (cuerpos y fuerzas de seguridad del Estado, investigadores privados, etc.), siempre que tengan superado el examen de acceso a la Universidad.

Durante el año 2002 la sección de Málaga celebra el décimo aniversario de su actividad docente a pleno rendimiento y satisfacción. Con este motivo, presentamos una valoración de estos diez años de docencia a través de un estudio que ha tenido una doble finalidad: La primera de ellas, mostrar la evolución del número de alumnos que han cursado estos estudios, así como su perfil y, la segunda, reflejar los resultados de una encuesta realizada a las promociones $3^{\mathrm{a}}$ a $7^{\mathrm{a}}$ correspondientes a los años 1997 al 2001. Los resultados de una encuesta similar realizada a las promociones $1^{\mathrm{a}} \mathrm{y}$ $2^{a}$ de los años 1995 y 1996 están publicados en el número 30 de este boletín de septiembre de 1997.

\section{Evolución del número de} alumnos y su perfil

\section{A. Evolución}

El inició de la actividad docente en Málaga fue muy exitoso, cubriéndose sobradamente las 120 plazas ofertadas (cupo máximo) para el primer curso. Durante los cursos 93/94 y 94/95 la Titulación quedó completamente implantada con los segundo y tercer cursos respectivamente. La primera promoción de alumnos obtuvo su titulación en 1995. Durante el actual tendrá su título la $8^{\mathrm{a}}$ promoción.

La evolución experimentada por esta titulación se expone a continuación a partir del número de alumnos de nuevo ingreso y del total de alumnos matriculados en los tres cursos.

En la figura número $1 \mathrm{se} \mathrm{ob-}$ serva claramente el proceso de consolidación de nuevos ingresos de alumnos en la sección de Málaga, que se encuentra muy próximo al número máximo permitido en el plan de estudios (120). Esta circunstancia coloca a Málaga a curso académico 2001/2002 ob- la cabeza de las cinco universidades andaluzas donde se imparte el título.

Por otro lado, el número total de alumnos, considerado a partir de la suma de los tres cursos en que se estructura la titulación, presenta un perfil semejante, siendo nuestra sección la que cuenta con un mayor número de alumnos matriculados en la actualidad. De hecho, Málaga cuenta con un 37,5\% más de alumnos (que en términos

\section{METODOLOGIA}

Durante el mes de febrero de 2002 se remitió a los titulados en Criminología de las promociones $3^{\circ}$ y $7^{\circ}$ (1997 a 2001) un cuestionario anónimo para que evaluaran la docencia recibida en la sección de Málaga y para que opinaran sobre la utilidad de los estudios realizados, su posible adecuación al mundo laboral y el interés por participar en futuras actividades dirigidas a los postgraduados en Criminología.

El tamaño de la población fue de 130 alumnos, y los cuestionarios cumplimentados y remitidos al Instituto por éstos fueron 24, lo que representa una tasa de respuesta de $12,5 \%$.

Todos los datos estadísticos relativos a la evolución del número de alumnos y su perfil han sido obtenidos de los ficheros que obran en la secretaria de Málaga del I.A.I.C.

Los datos relativos a las distintas secciones del I.A.I.C. han sido facilitados por las respectivas secciones. Hay que señalar que la sección de Cádiz posee dos sedes en la misma provincia, una en Jerez y otra en Los Barrios. Esta última inició su actividad en el curso 99/ 00 , lo cual explica el incremento del número de matriculados durante ese curso.

En los gráficos donde aparece información de otras secciones del I.A.I.C, la serie de datos comienza en el curso académico cuya información ha sido facilitada por la respectiva sección.

\section{Boletín Criminológico $N^{0} 58 \quad$ Marzo-Abril $2002 \quad$ Página 2}


absolutos se corresponde con 83 matriculados más) que la sección que le sigue inmediatamente después.

En la figura número $2 \mathrm{se} \mathrm{ob-}$ serva este proceso. En la lectura de dicha figura el lector debe tener en cuenta que en Málaga durante el curso académico 1992/ 1993 sólo se impartía docencia en el primer curso, y durante el curso 1993/1994 en primero y segundo. Esta circunstancia no hace comparable el número de alumnos de esos años en Málaga con el de las demás secciones que comenzaron a impartir la docencia en Criminología unos años antes. Esa es la razón que nos lleva a exponer los datos en el gráfico número 2 a partir de 1994/1995, curso en el que la Titulación en Málaga quedó completamente implantada. conviene señalar que desde la creación del Título de Experto hasta la actualidad se han producido 1.026 nuevos ingresos en la sección de Málaga, y se han registrado un total de 2.423 matrículas. En la actualidad el número total de Titulados en Criminología en Málaga es de 310 .

\section{B. Perfil del alumno.}

La distribución por sexos, siguiendo la tónica general de las demás titulaciones universitarias, da lugar a una presencia
Para finalizar este apartado

Figura 2: «Evolución número total de alumnos en el I.A.I.C.»

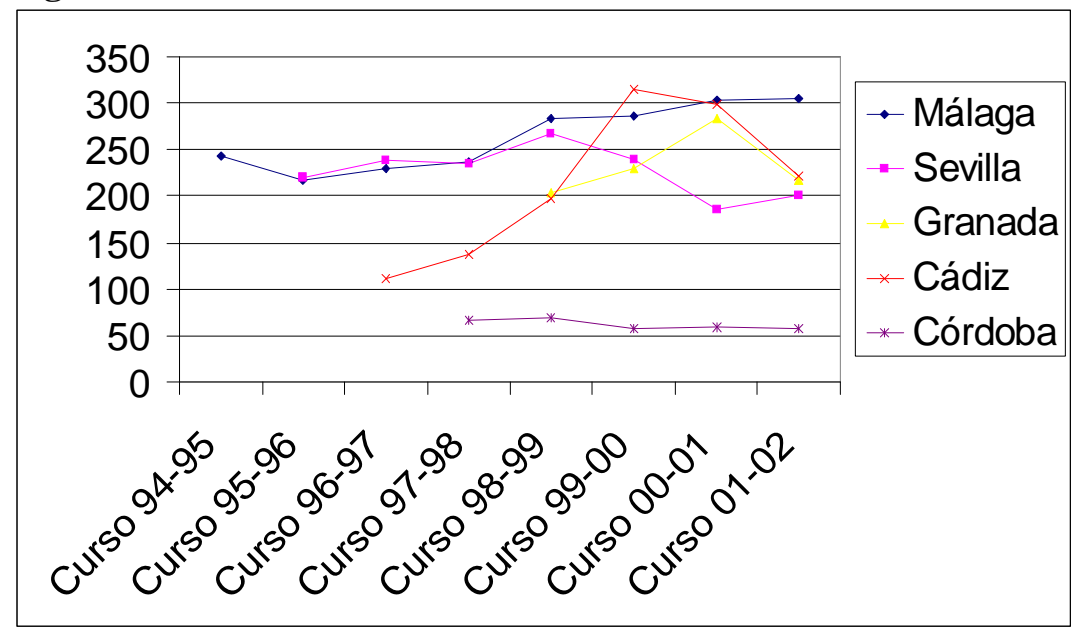

mayoritariamente femenina. En términos porcentuales y tomando como valor el número total de alumnos que se han matriculado en la titulación desde su creación, un $52 \%$ corresponde a alumnas y un $48 \%$ a alumnos.

Respecto a la distribución por sexos de los alumnos que están en posesión de la titulación, un 50,6\% es femenino frente a un $49,4 \%$ que es masculino.

La edad media de los alumnos se sitúa en el actual curso 2001/ 2002 en 31,58 años.

Respecto a la procedencia (considerada como la titulación o situación profesional desde donde se accede) es muy variada y heterogénea; sin embargo, desde el inicio de las actividades docentes de la sección de Málaga, la mayoría de nuestros alumnos pro-
Figura 3: «Procedencia de los alumnos»

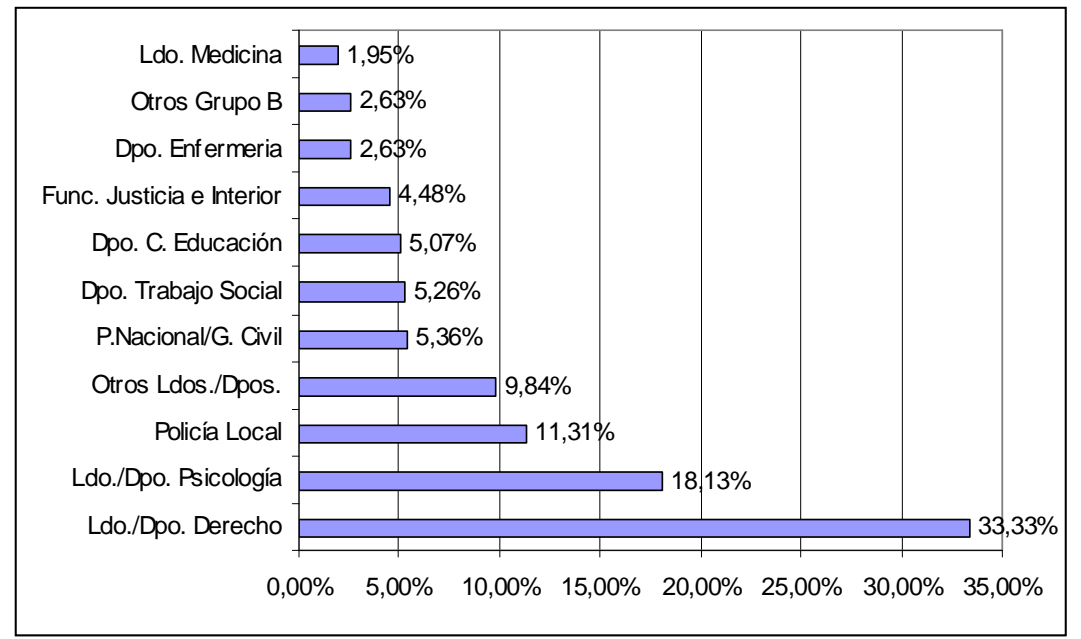

ceden de las licenciaturas de Derecho (con un total de 342 matriculados) y Psicología (con 186). Hay que destacar también el número de alumnos pertenecientes a la Policía Local que han accedido a nuestra titulación (116 en términos absolutos), el tercer grupo más numeroso. En la figura número 3 se muestra en términos relativos el origen tan variado de los alumnos que se han interesado por recibir una formación criminológica en la sección de Málaga. En el apartado de "otros licenciados o diplomados" se han incluido periodismo, relaciones laborales, informática, ciencias, etc, lo que muestra el interés que despierta esta materia en ámbitos de conocimiento muy diversos.

\section{Resultados de la encuesta}

\section{A. Ámbito de desarrollo pro-} fesional.

En la figura número 4 podemos observar las profesiones de los titulados en Criminología. Se distinguen cuatro grandes grupos: Cuerpos y Fuerzas de Seguridad con un $29,16 \%$, ejercicio libre de la abogacía con un $20,83 \%$, funcionarios de Instituciones Penitenciarias y Ministerio de Justicia e Interior con un $12,5 \%$ y sanidad con un $12,5 \%$. En un análisis comparativo con la encuesta de 1997 cabe destacar la aparición de este

\section{Boletín


último grupo de titulados que desarrollan su actividad dentro del ámbito de la sanidad.

A diferencia de otras titulaciones universitarias, el alumno que accede a Criminología desempeña mayoritariamente, con anterioridad a su acceso, una actividad profesional. Esa es la razón de la inexistencia de paro entre los encuestados que han respondido (7\% en la encuesta de 1997). Por otra parte, aunque el $70,83 \%$ respondió que la obtención del título no le ha reportado un ascenso profesional, el $29,41 \%$ considera que a corto o medio plazo conseguirá, gracias a los nuevos conocimientos adquiridos, una promoción profesional.

\section{B. Valoración de la Titulación.}

El contenido de las enseñanzas recibidas y la calidad didáctica del profesorado que las impartió les ha merecido a los encuestados (en una escala del 1 al 10) la siguiente puntuación:

\begin{tabular}{lc} 
Área & Puntuació \\
\hline Jurídica & 7,58 \\
Criminológica & 7,25 \\
Psicológica & 6,17 \\
Sociológica & 5,78 \\
Forense & 8,79 \\
Estadística & 5,81
\end{tabular}

Los seminarios impartidos en el Instituto han merecido una valoración de 7,5 (6,2 en la encuesta de 1997). A la pregunta de cuál destacaría como el más interesante y mejor realizado, para el $16,6 \%$ fueron los relativos a la asignatura de Medicina Legal, para un 12,5\% los de Policía Científica y para el $9,52 \%$ uno que se organizó sobre agresiones sexuales por el área de Psicología. En este apartado cabe destacar el mayor grado de satisfacción de los alumnos con los seminarios organizados en comparación con el de los titulados de la anterior encuesta, lo que hace que la valoración de los seminarios

Figura 4: «Ámbito de desarrollo profesional de los titulados»

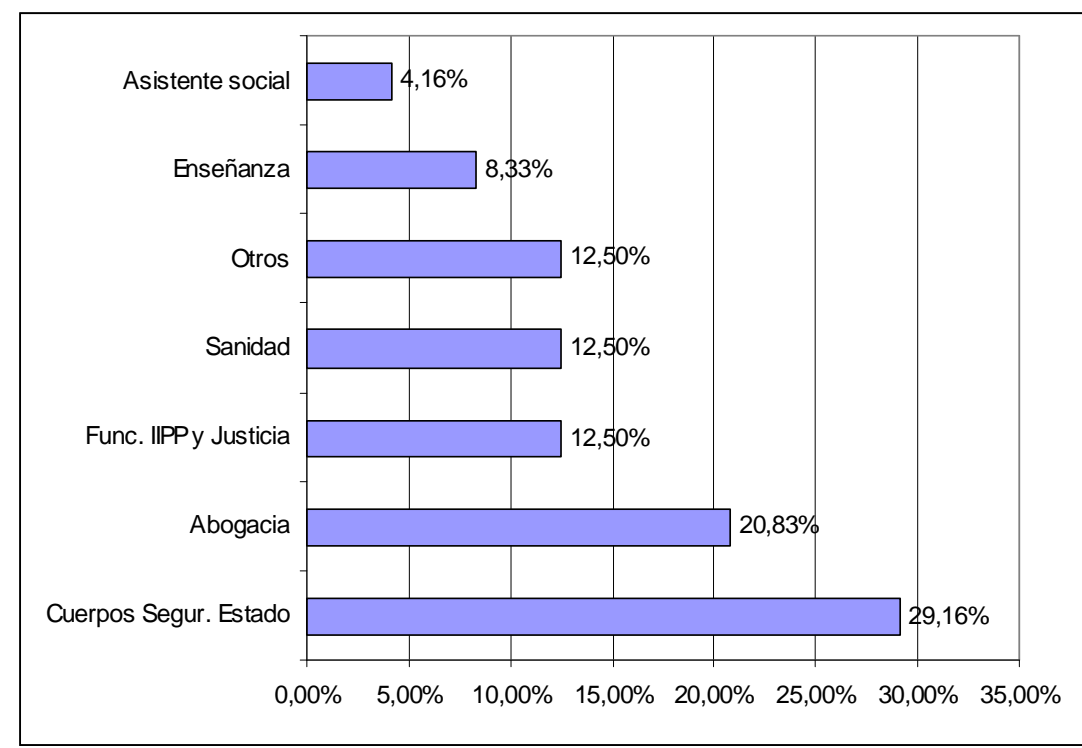

haya aumentado en más de un punto y que la respuesta a esta pregunta haya sido mucho más variada.

La expectativa inicial que tenía el alumno cuando ingresó en la sección de Málaga del I.A.I.C. ha sido satisfecha con una puntuación de 7,6 (6,2 en la encuesta de 1997).

Respecto a la atención administrativa recibida durante su estancia en el Instituto por el Personal de administración y servicios adscrito al I.A.I.C., se valora con 7,71 puntos (7,5 anteriormente).

El $65,22 \%$ considera que los materiales disponibles en la biblioteca son adecuados y el 8,69\% muy adecuados, mientras que el 26,09\% los considera poco adecuados.

Para finalizar este apartado sobre la titulación, los encuestados debían de valorar globalmente su paso por el I.A.I.C., al que califican con 7,4 puntos (7,6 en 1997).

\section{Actividades docentes pro- puestas.}

Los encuestados, al igual que en 1997, muestran un alto interés en que el Instituto de Criminología realice actividades donde su participación pueda tener cabida. En este apartado vamos a analizar las distintas propuestas realizadas.

A la pregunta de si estaría interesado en que el título organizara cursos de especialización para los postgraduados en Criminología sobre distintas materias, un $62,5 \%$ se muestra muy interesado. Por otro lado, se les pidió en el cuestionario que señalaran la materia sobre las que les gustaría recibir estos cursos especializados. Las más destacadas son las siguientes: $1^{\circ}$ Medicina Legal $(26,83 \%) ; 2^{\circ}$ Policía Científica $(24,39 \%) ; 3^{\circ}$ Políticas Preventivas $(21,95 \%) ; 4^{\circ}$ Criminología (13,07 \%).

Igualmente los encuestados muestran su interés en que el I.A.I.C. realice otras actividades donde puedan específicamente intervenir los titulados, destacando: prácticas $(33,3 \%)$; intercambios científicos $(18,3 \%)$; investigación $(16,6 \%)$; visitas a centros de interés $(13,3 \%)$; seminarios $(11,6 \%)$ y conferencias $(6,6 \%)$.

Por último, se les preguntó qué les gustaría incluir y eliminar en los estudios. Como respuesta más significativa destacamos la realización de más prácticas en la titulación, con un $41,6 \%$ de los encuestados.

\section{Boletín Criminológico $\quad N^{0} 58 \quad$ Marzo-Abril $2002 \quad$ Página 4}

